\title{
ON FK-SPACES WHICH ARE BOUNDEDNESS DOMAINS
}

\section{GLENN MEYERS}

ABSTRACT. An open question in summability theory is to characterize those $F K$-spaces, $E$, which are boundedness domains (i.e., $E=m_{A}$ for some infinite matrix $A$ ). As a partial solution to this problem we give necessary and sufficient conditions for an $F K$ space $E$, which has the $T$-sectional boundedness property, to be equal to $m_{A}$ for some row-finite $A$.

1. Introduction. An open question in summability theory is to characterize those $F K$-spaces, $E$, which are boundedness domains (i.e., $E=m_{A}$ for some infinite matrix $A$ ). In this paper we solve this problem in the case when $E$ has the $T$-sectional boundedness property $(T-A B)$ and $A$ is rowfinite. In addition we give a characterization of those $F K$-spaces, $E$, which are closed subspaces of $m_{A}$ for some row -finite matrix $A$.

2. Preliminary ideas and results. A $K$-space is a locally convex space of real or complex sequences such that the projection seminorm $p_{j}(x)=\left|x_{j}\right|$ is continuous for $j=1,2, \cdots$. An $F K$-s pace is a complete, metrizable $K$ space. If $E$ is an $F K$-space and a Banach space we say that $E$ is a $B K$ space. If the $F K$ topology of $E$ can be generated by the seminorms $\left\{p_{j}: j=1,2, \cdots\right\}$ and a single additional seminorm, $q$, we say that $E$ is an almost $B K$-space. A continuous seminorm, $q$, on an almost $B K$-space $E$ is said to be nontrivial if $q$ and $\left\{p_{j}: j=1,2, \cdots\right\}$ generate the topology of $E$.

Let $s$ be the set of all sequences and let $m$ be the set of all bounded sequences. $m$ is a $B K$-space with the norm $\|x\|_{\infty}=\sup _{k}\left|x_{k}\right|$. Let $A$ be an infinite matrix. Define $A x$ to be the sequence whose $i$ th term is $\sum_{k=1}^{\infty} a_{i k} x_{k}$ provided the series converges. For an $F K$-space $E$ and an infinite matrix $A$ we define $E_{A}=\{x \in s: A x \in E\} . \quad m_{A}$ is called the boundedness domain of $A$. If $A$ is row-finite, $m_{A}$ is an almost $B K$-space with its topology generated by the seminorms $\|A x\|_{\infty}$ and $\left\{p_{j}: j=1,2, \cdots\right\}$. See $[5, \S 12.4]$.

Presented to the Society, January 28, 1973; received by the editors August 13, 1973.

AMS (MOS) subject classifications (1970). Primary 40H05, 46A45, 46 A35. 
A sequence $x=\left(x_{k}\right)$ is said to be finite if $x_{k}=0$ for all but finitely many $k$. Let $E^{\infty}$ denote the set of finite sequences. Throughout this paper we will assume that every $F K$-space, $E$, contains $E^{\infty}$. Let $T=\left(t_{m n}\right)$ be a regular row-finite matrix. Let $e^{k}$ be the sequence with 1 in the $k$ th position and 0's elsewhere. Define the $m$ th $T$-section of a sequence $x$ to be the finite sequence

$$
T_{m} x=\sum_{n=1}^{\infty} t_{m n} \sum_{k=1}^{n} x_{k} e^{k}
$$

An FK-space $E$ has $T$ - $A K$ if $x=\lim _{m} T_{m} x$ with respect to the topology of $E$; for all $x \in E$. $E$ has $T-A B$ if the set of $T$-sections of $x$ is bounded for each $x \in E$. Unless otherwise stated, $E$ will denote an $F K$-space having $T-A B$. We denote by $E_{T-A K}$ the set $\left\{x \in E: x=\lim _{m} T_{m} x\right\}$. If $E$ has $T-A B$, $E_{T-A K}$ is a closed subspace of $E$. The $T-A K$ and $T-A B$ properties are discussed in [3] and [4].

Let $A$ be any coordinatew ise bounded subset of $s$. Define

$$
p_{A(T)}(x)=\sup _{m ; a \in A}\left|\sum_{k=1}^{\infty} a_{k}\left(T_{m} x\right)_{k}\right| \text {. }
$$

Let $F_{A(T)}=\left\{x \in s: p_{A(T)}(x)<\infty\right\}$. By [4, Theorem 4.2] $F_{A(T)}$ is an almost $B K$-space with its topology genereted by $p_{A(T)}$ and $\left\{p_{j}: j=1,2, \cdots\right\}$. If $A=\left(a_{i k}\right)$ is an infinite matrix, $A$ will be regarded as a countable set of sequences $\left\{a^{i}: a_{k}^{i}=a_{i k}\right\}$.

2.1. Proposition. If $A$ is row-finite and $m_{A}$ has $T-A B$ then $m_{A}=F_{A(T)^{\circ}}$

Proof. If $m_{A}$ has $T-A B$, then $p_{A(T)}(x)=\sup \left\|A\left(T_{m} x\right)\right\|_{\infty}<\infty$. Thus $m_{A} \subseteq F_{A(T)^{\circ}}$ Suppose $x \in F_{A(T)^{\circ}}$ Then

$$
\|A x\|_{\infty} \leq \sup _{i, m}\left|\sum_{n=1}^{\infty} t_{m n} \sum_{k=1}^{n} a_{i k} x_{k}\right|=p_{A(T)}(x)<\infty .
$$

Thus $F_{A(T)} \subseteq m_{A}$.

For any sequence space $E$, define

$$
E^{\gamma(T)}=\left\{y \in s: \sup _{m}\left|\sum_{k=1}^{\infty} y_{k}\left(T_{m} x\right)\right|<\infty \text { for all } x \in E\right\} \text {. }
$$

Clearly $E \subseteq E^{\gamma(T) \gamma(T)}$. If $E=E^{\gamma(T) \gamma(T)}$ then $E$ is called a $\gamma(T)$ space. By [4, Proposition 4.3] $F_{A(T)}$ is a $\gamma(T)$-space. As a corollary 
to the above proposition we have that if $m_{A}$ has $T \cdot A B$, then $m_{A}$ is a $\gamma(T)$ space. Properties of boundedness domains related to $\gamma(T)$-spaces, when $T$ is the identity matrix, have been studied in [1].

Thus, two necessary conditions for an $F K$-space $E$ with $T-A B$ to be a boundedness domain for some row-finite matrix are: (1) $E$ is an almost $B K$ space; and (2) $E$ is a $\gamma(T)$-space. We now turn to the task of proving them sufficient. But before we can do this we must further examine the structure of the space $F_{A(T)^{\circ}}$

3. The space $F_{A(T)}$. When $E$ is a $T-A K$ space, it follows from [4, Theorem 3.2] that $E^{\gamma(T)}$ can be identified with the dual space of $E$. If $E$ has $T-A B$ it then follows from [4, Theorem 3.3] that $E^{\gamma(T)}$ can be identified with the dual space of $E_{T-A K}$. If $E$ has $T-A B$, we can then give $E^{\gamma(T)}$ the weak ${ }^{*}$ topology induced by $E_{T \cdot A K}$. For a set $A \subseteq E^{\gamma(T)}$ we will denote the weak ${ }^{*}$ closed absolutely convex cover of $A$ by $(\Delta A)^{-}$.

3.1. Lemma. Let $E$ be an FK-space having $T-A B$. Then $p_{A(T)}(x)=$ $P_{(\Delta A)-(T)}(x)$ for all $x \in s$ and $A \subseteq E^{\gamma(T)}$.

Proof. Define the bilinear functional

$$
u(x, a)=\lim _{m} \sum_{k=1}^{\infty} a_{k}\left(T_{m} x\right)_{k}
$$

on $E_{T \cdot A K} \times E^{\gamma(T)} \cdot E_{T \cdot A K}$ and $E^{\gamma(T)}$ are in duality with respect to this bilinear functional and the weak ${ }^{*}$ topology is compatible with this duality. By [5, $\$ 13.2$, Theorem 1], $A^{00}=(\Delta A)^{-}$. It is easily verified that $\sup _{a \in A}|u(x, a)|=\sup _{a \in A} 00|u(x, a)|$ for all $x \in E_{T \cdot A K^{*}}$. Now for any $x \in s$, $T_{m} x \in E_{T \cdot A K}$. Thus

$$
p_{A(T)}(x)=\sup _{m ; a \in A}\left|u\left(T_{m} x, a\right)\right|=\sup _{m ; a \in A^{00}}\left|u\left(T_{m} x, a\right)\right|=p_{(\Delta A)^{-}(T)}(x) .
$$

As a result, we have that $F_{A(T)}=F_{(\Delta A)-(T)}$ for any $A \subseteq E^{\gamma(T)}$ 。

3.2. Lemma. $E^{\infty}$ is weak ${ }^{*}$ dense in $E^{\gamma(T)}$.

Proof. Suppose $x \in E_{T \circ A K}$ is such that $\lim _{m} \sum_{k=1}^{\infty} y_{k}\left(T_{m} x\right)_{k}=0$ for all $y \in E^{\infty}$. Since $T$ is regular, we must have that $\lim _{m}\left(T_{m} x\right)_{i}=x_{i}=0$ for all $i=1,2, \cdots$. Thus $x=0$. The result then follows from the Hahn-Banach theorem. 
4. Main results. Let $q$ be a continuous seminorm on $E$. Define

$$
B_{q}=\left\{y \in E^{\gamma(T)}: \sup _{x \in E_{T \cdot A K} ; q(x) \leq 1}\left|\lim _{m} \sum_{k=1}^{\infty} y_{k}\left(T_{m} x\right)_{k}\right| \leq 1\right\} .
$$

4.1. Theorem. Let $E$ be an FK-space which has $T-A B$.

(1) $E=m_{A}$ for some row-finite matrix $A$ if and only if $E$ is an almost $B K$-space and $E$ is a $\gamma(T)$-space.

(2) $E$ is a closed subspace of $m_{A}$ for some row-finite matrix $A$ if and only if $E$ is an almost $B K$-space and there is a number $M>0$ such that $q(x) \leq M \sup _{m} q\left(T_{m} x\right)$ for all $x \in E$, for some nontrivial seminorm $q$.

Proof. The proof of the necessity of (1) was given in $\$ 2$. Suppose $E$ is an almost $B K$-s pace and a $\gamma(T)$-space。 Let $q$ be a continuous nontrivial seminorm on $E$. By the construction in [4, Theorem 4.4] $F_{B}(T)=$ $E^{\gamma(T) \gamma(T)}=E$. By Lemma 3.2 there is a countable set of finite sequences, $A^{\prime}$, such that $\left(\Delta A^{\prime}\right)^{-}=B_{q}$. Let $A$ be the matrix with the set of rows equal to $\left\{T_{m} a: a \in A^{\prime}, m=1,2, \cdots\right\}$. Then $m_{A}=F_{A^{\prime}(T)}$ which, by Lemma 3.1, is equal to $F_{B_{q}(T)}$ which is in turn equal to $E$.

The necessity of (2) follows since $E$ has $T-A B$. To show the sufficiency we have from [4, Theorem 4.4] that $E \subseteq E^{\gamma(T) \gamma(T)}=m_{A} \cdot$ By $[5, \S 11.3$, Corollary 1] the $F K$ topology of $E$ is stronger than that of $m_{A}$. The hypothesis assures that it is weaker. Thus $E$ is closed in $m_{A}$.

As a special case we obtain that $l^{p}(1 \leq p \leq \infty)$ is a boundedness domain. This result was originally obtained by Bennett in [2, Proposition 9]. We point out that his proof is quite similar to the proof of Theorem 4.1.

4.2. Corollary. Let $E$ be an almost BK-space having T-AB. Suppose further that $E$ is a $\tau(T)$-space. Let $A$ be a row-finite matrix such that $E_{A}$ has $T-A B$. Then $E_{A}$ is a boundedness domain.

Proof. By Theorem 4.1, $E=m_{B}$ for some row -finite matrix $B$. Since $A$ and $B$ are both row-finite, it is easily verified that $E_{A}=m_{B A}$.

We remark that the hypothesis that $A$ is row-finite cannot be omitted from the above theorem. Let $A$ be the matrix with 1 's in the first row and 0 's elsewhere. Then $m_{A}=c s$, the set of convergent series, which is not a $\gamma(T)$-space.

In [6] an example of a convergence domain, $c_{A}$, is given in which $c_{A}$ does not have $T-A B$ for any regular matrix $T$ 。 It follows that $m_{A}$ does not 
have $T-A B$ for any regular $T$. Thus our results do not cover all possible boundedness domains for row-finite matrices.

\section{REFERENCES}

1. G. Bennett, Ph.D. thesis, Cambridge University.

2. - A new class of sequence spaces with applications in summability theory, J. Reine Angew. Math. (to appear).

3. M. Buntinas, On Toeplitz sections in sequence spaces (to appear).

4. G. Meyers, On Toeplitz sections in FK-spaces, Studia Math. 51 (to appear).

5. A. Wilansky, Functional analysis, Blaisdell, New York, 1964. MR 30 \#425.

6. A. Wilansky and K. Zeller, A biorthogonal system which is not a Toeplitz basis, Bull. Amer. Math. Soc. 69 (1963), 725-726. MR 27 \#1802.

DEPARTMENT OF MATHEMATICS, UNIVERSITY OF RHODE ISLAND, KINGSTON, RHODE ISLAND 02881 